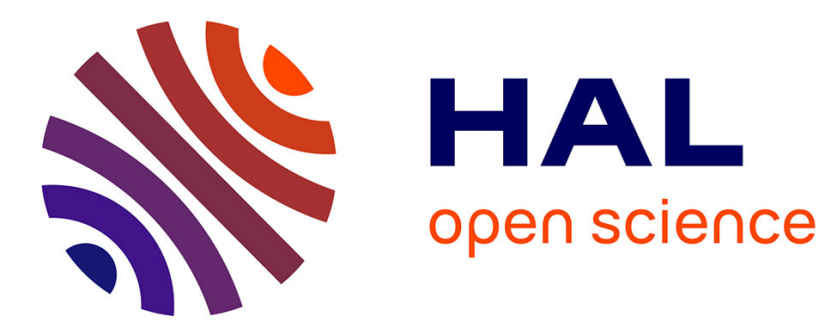

\title{
La traite mécanique des chèvres
}

\author{
J. Le Du
}

\section{- To cite this version:}

J. Le Du. La traite mécanique des chèvres. Productions Animales, 1989, 2 (1), pp.31-38. hal00895851

\section{HAL Id: hal-00895851 \\ https://hal.science/hal-00895851}

Submitted on 1 Jan 1989

HAL is a multi-disciplinary open access archive for the deposit and dissemination of scientific research documents, whether they are published or not. The documents may come from teaching and research institutions in France or abroad, or from public or private research centers.
L'archive ouverte pluridisciplinaire HAL, est destinée au dépôt et à la diffusion de documents scientifiques de niveau recherche, publiés ou non, émanant des établissements d'enseignement et de recherche français ou étrangers, des laboratoires publics ou privés. 
INRA Prod. Anim., 1989, 2 (1), 31-38

\section{J. $L E D U$}

INRA Laboratoire de Recherches sur la Traite

Domaine de la Motte-au-Vicomte

B.P. 29

35650 Le Rheu

\section{La traite mécanique des chèvres}

Traditionnellement, l'élevage caprin est considéré comme une activité marginale orientée vers la fabrication de fromages fermiers et nécessitant peu d'investissement. Or, on assiste à la disparition des petits troupeaux (moins de 20 chèvres traites à la main) compensée par la création de nouveaux élevages de 50 à 200 chèvres. Dans ces conditions plus intensives où l'atelier caprin constitue souvent l'activité principale de l'exploitation, la mécanisation de la traite doit obligatoirement être envisagée pour mieux gérer la main-d'œuvre et diminuer la pénibilité du travail.

Dans tous les cas, l'éleveur est confronté aux mêmes problèmes : choisir une installation de

\section{Résumé}

Avec une machine à traire, le nombre de chèvres traites par heure est indépendant de la vitesse de traite individuelle de chaque animal si l'on dispose d'un nombre suffisant de faisceaux-trayeurs. Mais, pour optimiser l'emploi de la maind'œuvre, il faut envisager de supprimer des interventions manuelles telles que le lavage du pis et l'égouttage, de moduler les intervalles entre traites voire de supprimer certaines d'entre elles, par exemple en trayant une fois par jour en fin de lactation. Les pertes de lait demeurent souvent acceptables et un trayeur habile peut traire, sans problème de mammites, jusqu'au 240 chèvres en une heure dans un manège bien conçu.

L'équipement de traite pour chèvres a été peu étudié. Une fréquence de pulsation de 70 à 100 pulsations par minute; un rapport de succion de 50 à $70 \%$ et un niveau de vide de 36 à $44 \mathrm{kPa}$ apparaissent justifiés. D'autres caractéristiques telles que la hauteur du lactoduc, le type de manchon, le poids du faisceau-trayeur et le volume de la griffe semblent avoir moins d'importance. Habituellement, le volume de la griffe et la capacité de la pompe à vide sont plus élevés que pour les vaches bien que les raisons ne soient pas clairement établies. traite plus ou moins mécanisée voire automatisée et définir une organisation rationnelle de son travail.

Pour diminuer le temps de travail et mieux l'organiser, il faut s'interroger sur l'utilité de certaines opérations. Faut-il laver le pis avant la traite? Pratiquer un égouttage après la traite? Distribuer un aliment pendant la traite? Faut-il adopter des intervalles de 12 heures entre traites? Peut-on supprimer certaines traites, par exemple le dimanche soir, ou traire une fois par jour en fin de lactation? Lorsqu'on a atteint un haut degré d'organisation du chantier de traite, le choix de la machine à traire proprement dit revêt une importance particulière. En effet, l'obtention d'une bonne traite, avec une vidange du pis rapide et complète, sans traumatisme des trayons et sans problème de mammites, dépend en grande partie de la conception des composants de la machine à traire et du réglage de ses paramètres de fonctionnement.

\section{1 / Les installations de traite}

Depuis 1974, plusieurs enquêtes ont été conduites en France par l'ITOVIC, qui proposent des plans types d'installation. Un bon aperçu des conditions de traite obtenues est donné par Disset (1974), Le Mens et al (1978) et Le Jaouen (1981).

\section{1 / La traite en chèvrerie}

Il est possible de monter une installation de traite mécanique dans la chèvrerie. En général, une plate-forme de $0,80 \mathrm{~m}$ à $1 \mathrm{~m}$ de haut est 
construite pour recevoir les animaux qui accèdent aux cornadis, attirés par une distribution d'aliments concentrés. La traite se fait le plus souvent en bidons car l'installation d'un lactoduc est rarement envisageable dans une chèvrerie. La traite en chèvrerie présente quelques avantages. Elle évite la construction d'une salle de traite et peut conduire à un gain de temps car la circulation des animaux est diminuée. Par contre, la traite s'effectue dans des conditions peu hygiéniques qui peuvent nuire à une bonne fabrication fromagère. De plus le trayeur travaille dans des conditions inconfortables sur du fumier et au milieu des animaux. Pour travailler dans de bonnes conditions, il faut aménager une salle de traite équipée de quais de traites (installations statiques) ou d'un manège de traite.

\section{2 / Les quais de traite}

Il existe quatre types de quais surélevés par rapport au sol (figure 1). De multiples variantes sont possibles pourvu que l'on respecte les règles suivantes :

Figure 1. Les principaux types de quais de traite (d'après Le Du 1987).

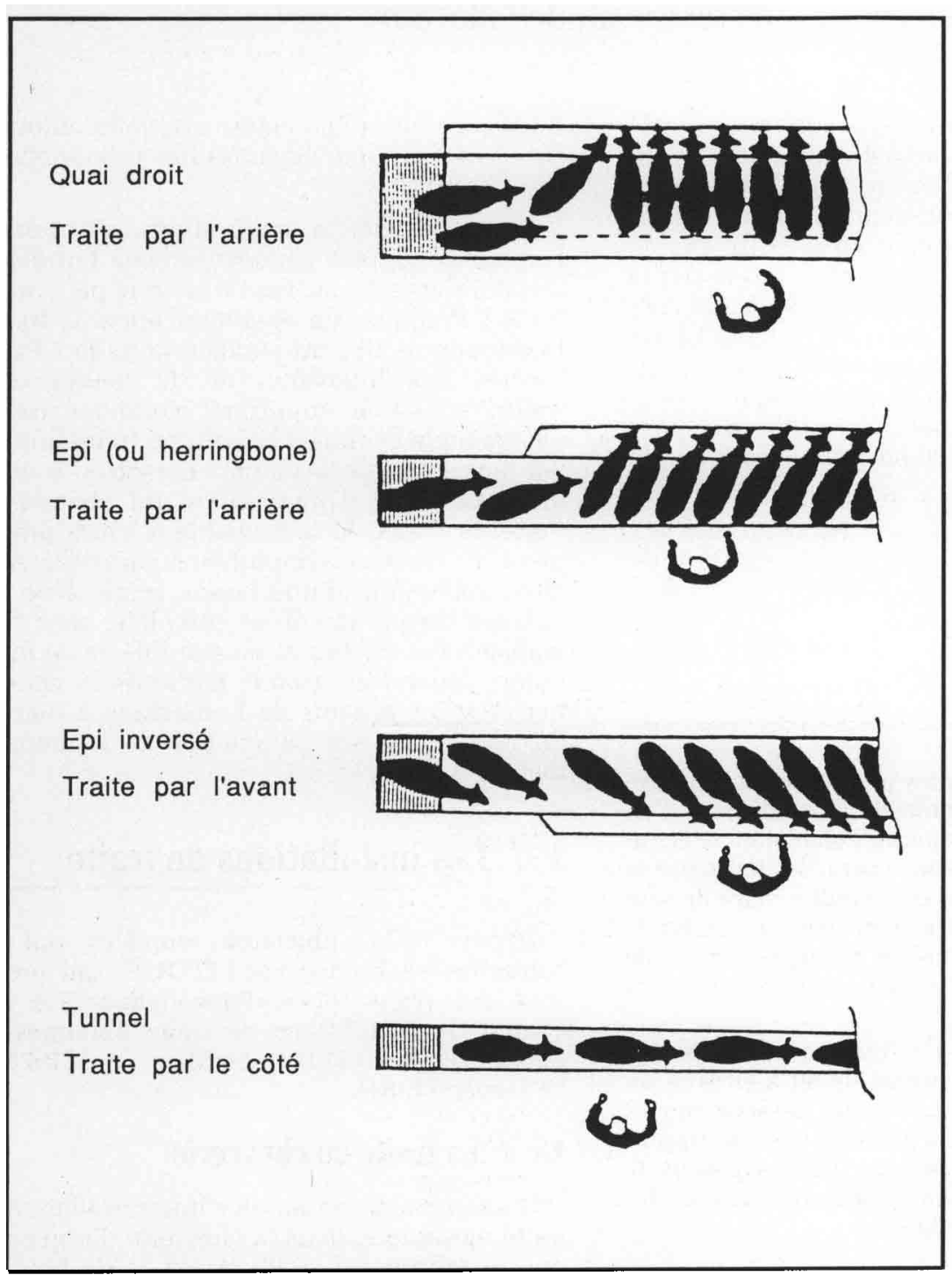

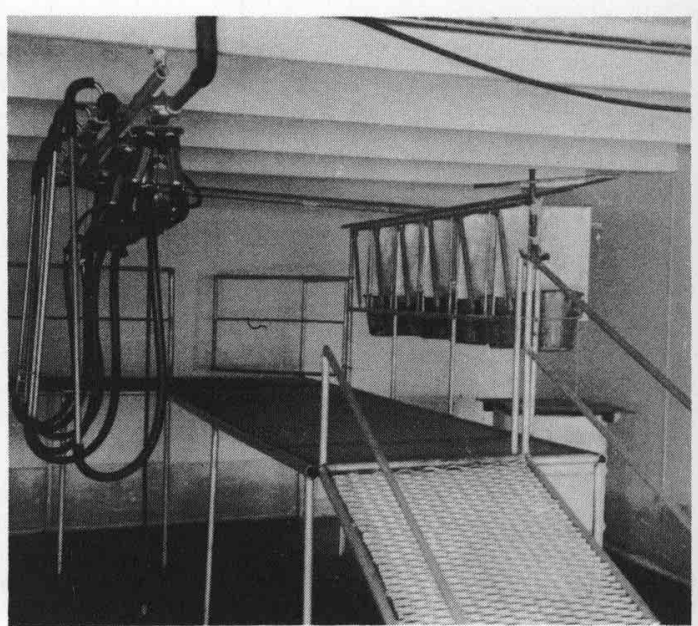

L'équipement de traite peut être très simple. Ici, un quai droit pour 6 chèvres.

- les chèvres sont en général bloquées au niveau de la tête dans un cornadis et une distribution de concentrés peut être effectuée (sauf avec un tunnel),

- le nombre de stalles sur un quai, limité à 4 ou 5 avec un tunnel, peut atteindre 24 avec les autres quais,

- au lieu d'avoir un seul quai (quai simple), on peut disposer 2 quais côte à côte (quai double). Ils sont en général séparés par une fosse où se tiennent les trayeurs,

- un trayeur peut utiliser 2 à 8 faisceauxtrayeurs. L'équipement pourra être d'un faisceau-trayeur par chèvre lorsque le nombre de stalles est faible. Dans les grandes installations, il est courant d'utiliser un faisceau pour 2 chèvres ou même un faisceau pour 4 chèvres.

Avec la traite mécanique, le nombre de chèvres traites par heure peut être indépendant de la vitesse de traite et de la production laitière des animaux si le nombre de faisceaux-trayeurs est suffisant. Celui-ci doit donc être choisi après mûre réflexion car il conditionne le nombre de chèvres que l'on pourra traire par homme et par heure.

Les enquêtes montrent que les productivités sont extrêmement variabes. Selon, Le Mens (1981) un homme trait par heure entre 23 et 107 chèvres pour des productions journalières de 1,7 à 3,0 litres de lait. Le tableau 1 montre qu'il n'existe pas de relation nette entre l'équipement dont dispose le trayeur (nombre de stalles et de faisceaux) et la productivité. En fait, nous verrons que les productivités les plus élevées sont atteintes seulement si l'on choisit de simplifier voire de supprimer certaines interventions manuelles du trayeur telles que le lavage avant la traite (préparation) et l'égouttage après la traite.

\section{3 / Les manèges de traite}

Dans les salles de traite rotatives, appelées " manèges de traite", les chèvres et les faisceaux trayeurs sont portés par une plate-forme mobile en rotation. Il existe de nombreux types conçus selon les mêmes principes que pour les 
Tableau 1. Performances observées dans différents chantiers de traite selon les résultats d'une enquête BCMEA et ITOVIC de 1976 cités par Le Mens (1983).

\begin{tabular}{|c|c|c|c|c|c|}
\hline & $\begin{array}{l}\text { Nombre } \\
\text { de places }\end{array}$ & $\begin{array}{c}\text { Nombre } \\
\text { de faisceaux }\end{array}$ & $\begin{array}{c}\text { Nombre } \\
\text { de faisceaux } \\
\text { par trayeur }\end{array}$ & $\begin{array}{l}\text { Lait par jour } \\
\text { et par chèvre } \\
\text { (litres) }\end{array}$ & $\begin{array}{c}\text { Nombre } \\
\text { de chèvres } \\
\text { par trayeur } \\
\text { par heure }\end{array}$ \\
\hline $\begin{array}{c}\text { Traite } \\
\text { manuelle }\end{array}$ & & & & $\begin{array}{l}2,4 \\
2,1\end{array}$ & $\begin{array}{l}22 \\
23 \\
31 \\
37\end{array}$ \\
\hline $\begin{array}{c}\text { En } \\
\text { chèvrerie }\end{array}$ & & $\begin{array}{l}2 \\
2 \\
5 \\
6 \\
4\end{array}$ & $\begin{array}{l}2 \\
2 \\
5 \\
6 \\
4\end{array}$ & $\begin{array}{l}2,7 \\
2,5 \\
3,0 \\
2,6 \\
2,0\end{array}$ & $\begin{array}{r}59 \\
61 \\
\mathbf{8 8} \\
94 \\
107\end{array}$ \\
\hline $\begin{array}{l}\text { Quai } \\
\text { simple }\end{array}$ & $\begin{array}{r}12 \\
15 \\
16 \\
24 \\
8 \\
10 \\
8\end{array}$ & $\begin{array}{r}12 \\
4 \\
4 \\
6 \\
4 \\
6 \\
4\end{array}$ & $\begin{array}{c}4,3 \\
2 \\
3,2 \\
2 \\
4 \\
6 \\
4\end{array}$ & $\begin{array}{l}2,8 \\
3,1 \\
3,0 \\
2,8 \\
2,3 \\
2,3 \\
2,8\end{array}$ & $\begin{array}{l}22 \\
24 \\
37 \\
40 \\
44 \\
43 \\
62\end{array}$ \\
\hline $\begin{array}{c}\text { Quai } \\
\text { double }\end{array}$ & $\begin{array}{r}20 \\
50 \\
28 \\
20 \\
8 \\
8 \\
28 \\
8\end{array}$ & $\begin{array}{r}4 \\
6 \\
14 \\
10 \\
4 \\
8 \\
14 \\
8\end{array}$ & $\begin{array}{c}2 \\
2,5 \\
7 \\
5 \\
4 \\
8 \\
7 \\
8\end{array}$ & $\begin{array}{l}2,5 \\
2,4 \\
2,5 \\
\\
2,5 \\
2,2 \\
2,5 \\
2,2\end{array}$ & $\begin{array}{r}23 \\
34 \\
40 \\
41 \\
55 \\
72 \\
73 \\
102\end{array}$ \\
\hline Manège & $\begin{array}{r}18 \\
5 \\
5 \\
8 \\
8\end{array}$ & $\begin{array}{l}9 \\
5 \\
5 \\
8 \\
8\end{array}$ & $\begin{array}{c}4,5 \\
2,5 \\
2,5 \\
4 \\
8\end{array}$ & $\begin{array}{l}1,9 \\
2,6 \\
2,0 \\
2,2 \\
1,7\end{array}$ & $\begin{array}{l}44 \\
51 \\
53 \\
58 \\
78\end{array}$ \\
\hline
\end{tabular}

vaches (Le Mens 1977) permettant tous les modes de traite possibles : par l'arrière, par le côté, par l'avant. Lorsque le manège de traite est apparu en France vers 1970, il était le plus souvent conçu et construit par l'éleveur pour un faible coût. Actuellement, plusieurs constructeurs proposent des manèges de traite. Pour un seul trayeur, ils comportent de 4 à 8 stalles avec un faisceau par stalle. D'autres configurations sont possibles. On peut par exemple, avoir 10 à 12 stalles avec un faisceau et 2 trayeurs ou un faisceau pour 2 stalles et un seul trayeur.

Les productivités réelles sont très variables: de 40 à 130 chèvres traites par homme et par heure (Le Mens 1977). Il faut noter que les rendements les plus faibles sont souvent obtenus dans des manèges conçus pour un seul trayeur et utilisés par deux (tableau 1). En fait, lorsque l'installation est parfaitement conçue, la productivité peut être très élevée. Le Jaouen et al (1984) cite le cas d'un manège à 8 stalles avec un diamètre intérieur de $2,1 \mathrm{~m}$ où se trouve le trayeur qui posséde une console pour contrôler les mouvements du manège. Sans lavage du pis et sans égouttage, des essais dans 3 fermes différentes ont conduit à des productivités de 176, 221 et 356 chèvres traites par heure. Actuellement, un manège de traite bien conçu avec un minimum d'automatismes semble être la meil- leure solution pour traire dans des conditions intensives. Un trayeur expérimenté peut traire par heure 240 chèvres donnant 3,5 litres de lait par jour s'il utilise une technique de traite simplifiée aux maximum.

\section{2 / Les techniques de traite et l'organisation du travail}

Les possibilités de simplifier les techniques de traite et de moduler les horaires ont été l'objet de nombreuses études chez la vache et la brebis laitières. En ce qui concerne la chèvre, il existe peu de travaux fiables et nous serons souvent conduits à décrire les situations observées en ferme.

\section{1 / Le lavage du pis}

Outre son rôle hygiénique, le lavage du pis crée un massage de la glande susceptible de déclencher un réflexe d'éjection du lait tel qu'on l'observe chez la vache. Or, dans un essai sur 78 chèvres, Ricordeau et Labussière (1970) n'ont détecté aucun effet du lavage du pis sur le temps de traite et la production de lait. Chez la
Le nombre de chèvres traites par personne et par heure est très variable mais il n'existe pas de relation nette entre cette productivité et I'équipement dont dispose le trayeur. 


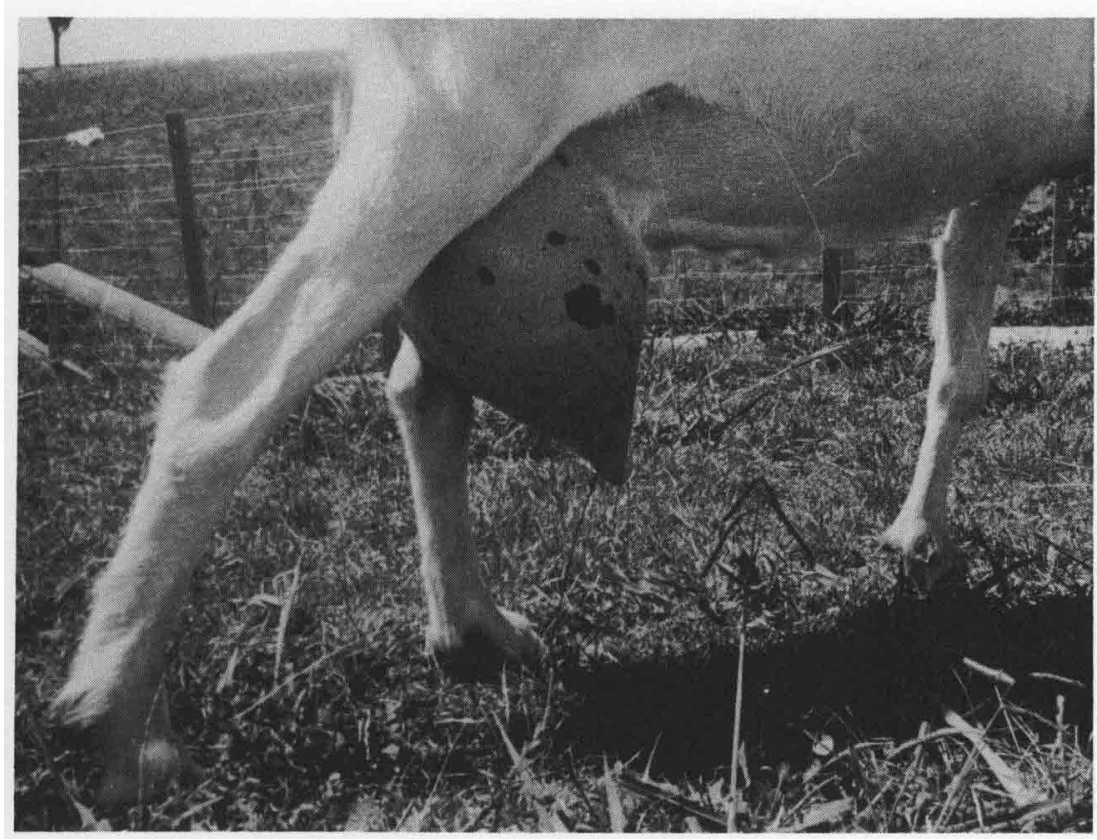

Chez la chèvre, le lait est en grande partie stocké dans la citerne, proportionnellement plus volumineuse que chez la vache.

chèvre, il semble que le fait de poser les gobelets trayeurs sur le pis soit suffisant pour stimuler le réflexe d'éjection du lait. Les mêmes résultats sont obtenus chez les brebis laitières qui sont toujours traites sans massage préalable de la mamelle (Labussière et al 1978).

Le Mens (1984) observe qu'un lavage biquotidien de la mamelle, en diminuant la résistance de la peau aux agressions microbiennes, peut accroître les problèmes sanitaires et en particulier les risques de mammites. Le lavage du pis, qui est une opération coûteuse en main-d'œuvre, devrait pouvoir être pratiqué de façon occasionnelle lorsque la mamelle est sale.

\section{2 / L'égouttage}

En fin de traite, l'égouttage machine consiste à pratiquer une traction sur la griffe ou les gobelets tout en massant le pis pour le vidanger complètement. Outre le fait d'obtenir plus de lait, on considère souvent que cette manipulation du pis est un bon moyen de surveiller l'état sanitaire et implicitement de prévenir les mammites.

Le volume de lait obtenu par égouttage est très variable d'un troupeau à un autre : de 40 à $200 \mathrm{ml}$ par chèvre selon Le Mens (1981). Il peut représenter une fraction importante de la production totale : $18 \%$ selon Ricordeau et Labussière (1970). En outre, il dépend beaucoup de la conception de certains composants de la machine à traire (manchon-trayeur) ainsi que du choix de certains paramètres (rapport de pulsation). On conçoit donc qu'il est d'autant plus facile de supprimer l'égouttage que ces facteurs de variation sont mieux maîtrisés.

De nombreuses études chez la vache montrent que la suppression complète de l'égouttage réduit la production laitière de $3 \%$ en moyenne et qu'il n'y a pas d'effets nocifs sur le plan sanitaire. Quelques travaux chez la chèvre conduisent à des conclusions similaires. Dans des conditions de traite où le volume d'égouttage représente $14 \%$ du lait total, lorsqu'on supprime l'égouttage on observe seulement une réduction de $3,4 \%$ de la production de lait, aucun effet sensible sur la quantité de matière sèche utile et la composition du lait, aucun effet sanitaire nocif (Le Mens 1984). Chez la chèvre, le lait extrait lors de l'égouttage semble être du lait citernal qui peut en partie demeurer dans la mamelle après la traite sans avoir pour effet de freiner la sécrétion. En 1977, selon Le Mens (1984), les trois quarts des éleveurs avaient supprimé cette opération car pour traire vite, il est indispensable d'habituer les chèvres à être traites sans égouttage.

\section{3 / Les intervalles entre les traites}

Chez la vache, de nombreuses études ont visé à comparer des intervalles égaux et inégaux entre les traites (Dodd et Griffin 1977). Les résultats montrent qu'avec des intervalles tels que 16 heures et 8 heures, il n'y a pas de baisse significative des productions de lait et de matières grasses même avec des animaux produisant 6000 litres de lait.

Chez la chèvre, une seule étude (Henderson et al 1983) montre qu'il n'existe pas de différences entre des intervalles égaux et inégaux (16 et 8 heures). Il est en général reconnu que la chèvre est facile à traire car une forte proportion du lait est stockée dans la citerne qui est proportionnellement plus volumineuse que chez la vache. Aussi, il apparaît peu probable que des intervalles inégaux entre les traites entraînent plus de problèmes chez la chèvre que chez la vache. En pratique, cette possibilité de moduler les horaires de travail est très souvent adoptée pour faciliter l'organisation du travail.

\section{4 / La fréquence des traites}

On accroît la production de lait lorsqu'on augmente le nombre de traites journalières. Toutefois, dans des conditions intensives, l'éleveur peut être plus intéressé par une réduction de la fréquence des traites, qui permet de libérer la main-d'œuvre nécessaire pour d'autres travaux, au moins à certaines périodes de l'année. Sur un troupeau de chèvres de race Alpine étudié pendant 7 ans à l'INRA, en trayant une seule fois par jour durant toute la lactation, Mocquot (1978 et 1980 ) a observé une réduction de la production de lait de 35 à $40 \%$ (de 223 à $290 \mathrm{~kg}$ ) et un raccourcissement de la lactation de 2 semaines. En commençant à traire une fois par jour après le deuxième mois de lactation, la production de lait est réduite de $20 \%(122 \mathrm{~kg})$ et la lactation de 10 jours environ, sans que la composition du lait soit affectée. Bien que la question n'ait pas été étudiée de façon approfondie, la suppression d'une traite par jour vers la fin de la lactation pourrait être une solution satisfaisante dans certaines conditions d'élevage. Ainsi, Mocquot (1978) cite un élevage où la perte de $10 \%$ seulement est compensée par une augmentation de l'effectif pour maintenir le revenu avec des conditions de travail plus agréables. 
Sur le même troupeau de l'INRA, la suppression de la traite du dimanche soir, pendant toute la lactation réduit la production de lait de 4,2 à $4,6 \%$ et celle des matières grasses de 2,8 à $3,0 \%$. Ces résultats sont confirmés par des observations faites dans des élevages avec des niveaux de production de l'ordre de $500 \mathrm{~kg}$ (Le Mens 1978).

En commençant à supprimer la traite du dimanche soir après un mois, deux mois ou cinq mois de lactation, les productions laitières sont réduites dans des proportions qui demeurent acceptables: respectivement 4,5\%, 3,1\% et $1,2 \%$. Aucun des systèmes expérimentés n'a conduit à une dégradation de l'état sanitaire. Toutefois, la suppression systématique ou occasionnelle de la traite du dimanche a pour conséquence de modifier dans les jours suivants la production et la composition du lait, qui redeviennent normaux seulement le mercredi soir ou jeudi matin. Ceci constitue un inconvénient pour la fabrication fermière de fromage. En outre, selon Mocquot (1980), «il faut insister sur l'inconvénient majeur que présente l'adoption non maîtrisée de ces pratiques dans les élevages adhérents au contrôle laitier. Actuellement, aucun dispositif ne permet de prendre en compte ces différentes options pour le calcul des lactations et l'indexation des reproducteurs ».

\section{3 / L'efficacité de la machine à traire}

Le matériel de traite s'inspire de celui utilisé pour les vaches ou pour les brebis laitières car les aspects spécifiques à la chèvre ont été peu étudiés. D'une part, on a toujours considéré que la traite des chèvres pose moins de problèmes que celle des vaches et des brebis, d'autre part, les industriels, considérant la production caprine comme marginale hésitaient à investir dans un marché limité.

\section{1 / La pulsation}

Il est possible de traire les chèvres dans les mêmes conditions que les vaches à la fréquence de pulsation de 60 pulsations par minute (p/min) (Le Du et Benmederbel, 1984) mais, selon certains physiologistes, des fréquences plus élevées seraient justifiées pour intensifier le réflexe d'éjection du lait (Grachev 1953 ; Tverskoi et al 1984). Chez la brebis au moins, de nombreuses comparaisons entre 60 $\mathrm{p} / \mathrm{min}$ et $180 \mathrm{p} / \mathrm{min}$ pendant des lactations complètes montrent les avantages d'une fréquence élevée quelles que soient les conditions expérimentales: race de brebis, méthode de traite, type de machine, etc... (Le Du 1985). Avec la fréquence la plus élevée de pulsation, une vidange plus complète du pis conduit à des accroissements significatifs de la production de lait et de matières grasses (tableau 2). En comparant 120 et $180 \mathrm{p} / \mathrm{min}$ sur deux lots de 115 brebis pendant une lactation complète, Casu et Carta (1974) ont observé, à $180 \mathrm{p} / \mathrm{min}$, un accroissement de la production de lait $(2,9 \%)$, de la quantité de matières grasses $(3,4 \%)$ et de celle de matières azotées $(5,1 \%)$. En contrepartie, les fréquences très élevées présentent des inconvénients : nécessité de pulsateurs électroniques avec relais électromagnétiques, accroissement du bruit, risque d'engorgement du faisceau par le lait pour des débits importants. Au vu de nos connaissances actuelles, il semble qu'une fréquence comprise entre 70 et 100 $\mathrm{p} / \mathrm{min}$ représente un bon compromis pour les chèvres dans les conditions françaises (tableau 3).

\section{LES DIFFÉRENTS PARAMÈTRES DE FONCTIONNEMENT DE LA MACHINE A TRAIRE}
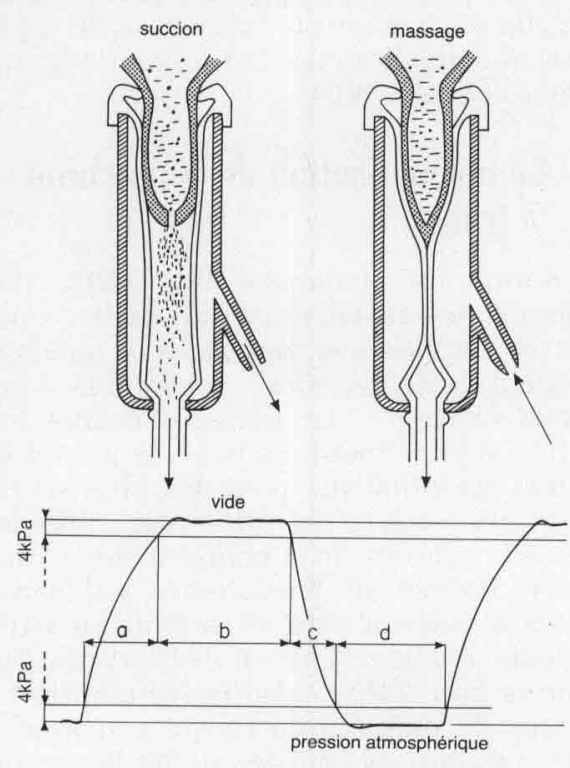

On distingue deux phases du fonctionnement du manchon-trayeur:

- Durant la phase de succion, la même dépression appelée " vide de traite », existe à l'intérieur et à l'extérieur du manchon. Celui-ci est ouvert et le lait s'écoule par le canal du trayon.

- Durant la phase de massage, de l'air à la pression atmosphérique est admis dans la chambre de pulsation qui entoure le manchon. Celui-ci est fermé et l'écoulement du lait cesse.

L'enregistrement des variations de pression dans la chambre de pulsation représente la « courbe de pulsation » définie par:

- Le vide de traite : valeur du vide atteinte durant la phase de succion (en $\mathrm{kPa}$ )

- La fréquence de pulsation : nombre de pulsations par minute (en $\mathrm{p} / \mathrm{min})$

- Le rapport de pulsation : $\frac{a+b}{a+b+c+d} \times 100$ (en \%)

En pratique, il est courant de ne considérer que deux phases :

$\mathrm{a}+\mathrm{b}:$ succion

$\mathrm{c}+\mathrm{d}$ : massage

Anciennement, le rapport de pulsation était défini de façon plus ou moins précise par une fraction : succion/massage (exemple : $3 / 1$ correspond à un rapport de $75 \%$ ). 
Tableau 2. Comparaison de deux fréquences de pulsation chez la brebis laitière : 180 et 60 pulsations par minute (Le Du 1985).

\begin{tabular}{|c|c|c|c|}
\hline & \multicolumn{2}{|c|}{$\begin{array}{l}\text { Différences entre les productions } \\
\text { à } 180 \mathrm{p} / \mathrm{min}(\mathrm{A}) \text { et } 60 \mathrm{p} / \mathrm{min}(\mathrm{B}) \\
\frac{\mathrm{A}-\mathrm{B}}{\mathrm{A}} \times 100 \mathrm{en} \%\end{array}$} & \multirow[t]{2}{*}{$\begin{array}{c}\text { Niveau } \\
\text { de signification }\end{array}$} \\
\hline & Moyennes & Valeurs extrêmes & \\
\hline Lait total (l) & $+2,7$ & $0,0 \grave{a}+7,2$ & $0,04(a)$ \\
\hline Lait machine (l) & $-0,3$ & $-7,0 \grave{a}+5,3$ & ns (a) \\
\hline Egouttage machine (l) & $+19,1$ & $-0,6$ à $+27,1$ & $0,02(b)$ \\
\hline Matières grasses totales $(\mathrm{kg})$ & $+6,0$ & $+1,7$ à $+10,3$ & $0,04(\mathrm{c})$ \\
\hline
\end{tabular}

* Nombre de brebis $\times$ traites pour chacun des 2 traitements :

(a) 85000 en 6 essais

(b) 51600 en 4 essais

(c) 49600 en 4 essais

Comme pour les vaches, le rapport de pulsation est le principal facteur déterminant le débit d'écoulement du lait qui croît de $23 \%$ lorsqu'on passe d'un rapport de pulsation de 50 à $75 \%$. Toutefois, bien que la production de lait ne soit pas affectée, la vidange du pis est plus difficile puisque le volume d'égouttage croît de $27 \%$ (Ricordeau et Labussière 1970). En outre, comme certaines observations montrent que les rapports les plus élevés pourraient dégrader l'état sanitaire du pis (mammites), les constructeurs tendent actuellement à éviter les rapports de pulsation supérieurs à $70 \%$ (Darracq et al 1978). Des valeurs comprises entre 50 et $70 \%$ de succion semblent raisonnables (tableau 3).

Tableau 3. Recommandations pour le choix des paramètres de fonctionnement.

Niveau de vide

36 à $44 \mathrm{kPa}$

Fréquence de pulsation

70 à $100 \mathrm{p} / \mathrm{min}$

Rapport de pulsation

50 à $70 \%$

\section{2 / Le niveau de vide}

Un accroissement du niveau de vide conduit à une diminution des temps de traite mais représente un risque de prédisposer l'animal aux mammites. Aussi, pour la chèvre, les constructeurs adoptent des niveaux de vide allant de 36 à $44 \mathrm{kPa}$, légèrement plus faibles que pour la vache et similaires à ceux qui sont utilisés pour la brebis (tableau 3).

Parfois, un double vide est utilisé afin d'accroître l'amplitude du mouvement du manchon. Dans ce cas, le vide de pulsation (espace annulaire du gobelet) est plus élevé que le vide de traite (intérieur du manchon). En pratique, ce système ne présente aucun avantage que ce soit pour les chèvres (Le Mens et al 1984) ou pour les brebis (Le Du et al 1978). Par contre, il existe très probablement un risque d'endommager les tissus du trayon et de déclencher des mammites.

\section{3 / Le faisceau-trayeur}

En général, les chèvres sont traites avec un faisceau similaire à celui des vaches : une griffe avec un grand volume intérieur et un orifice d'admission d'air. Parfois, des faisceaux conçus pour les brebis sont utilisés : griffe peu volumineuse sans admission d'air. En comparant des équipements très différents (poids de 0,3 à 1,4 $\mathrm{kg}$; volume de griffe de 0 à $140 \mathrm{ml}$ ), on observe des effets significatifs sur les débits d'écoulement du lait et les volumes d'égouttage (Cicogna et Sangiorgi 1984). Par contre, lorsqu'on compare des équipements spécialement conçus pour les chèvres, les différences sont peu marquées: $10 \%$ sur les débits en comparant six manchons du commerce (Bouillon et Le Mens 1984 ) ; aucun effet de la longueur du manchon (Le Du et Benmederbel, non publié) et aucun effet du volume de la griffe compris entre 40 et $350 \mathrm{ml}$ (Le Mens et Disset 1984). Pour les chèvres, une caractéristique étonnante de l'équipement est l'utilisation d'une griffe souvent posée sur le sol ayant un volume plus élevé que pour les vaches. Il n'existe aucun argument objectif qui justifie une telle conception de la griffe qui accroît les temps de manipulation du faisceau lorsqu'elle est très lourde (plus de $1700 \mathrm{~g}$ pour $350 \mathrm{ml}$ ) et n'améliore pas la finition de la traite (Le Mens et Disset 1984).

\section{4 / La normalisation de la machine à traire}

La norme internationale (ISO 5707, 1982) s'applique aux installations de traite «pour vaches et bufflesses». Les aspects qualitatifs concernent également les installations «pour brebis et chèvres ». La dernière norme française (U - 36011, 1985) est la seule à fixer des exigences quantitatives pour les chèvres. Par rapport aux vaches, la principale différence concerne la capacité de la pompe à vide et donc la réserve de vide de l'installation (tableau 4). La normalisation doit être fondée sur la connaissance scientifique et l'observation des pratiques courantes. Malheureusement, il existe peu de données en ce qui concerne les chèvres. Les raisons pour lesquelles la pompe à vide doit être plus puissante que pour les 
Tableau 4. Choix de la pompe à vide selon la norme française $U-36011$ (1985) pour les vaches et pour les chèvres.

\begin{tabular}{|cll|}
\hline \multicolumn{2}{|c|}{ Traite en pots } \\
Vaches & $\mathrm{C}=50+60 \mathrm{n}$ & $\mathrm{n} \leqq 10$ \\
& $\mathrm{C}=650+45(\mathrm{n}-10)$ & $\mathrm{n}>10$ \\
Chèvres & $\mathrm{C}=50+80 \mathrm{n}$ & $\mathrm{n} \leqq 10$ \\
& $\mathrm{C}=850+40(\mathrm{n}-10)$ & $\mathrm{n}>10$ \\
\hline Traite en lactoduc & \\
Vaches & $\mathrm{C}=150+60 \mathrm{n}$ & $\mathrm{n} \leqq 10$ \\
& $\mathrm{C}=750+45(\mathrm{n}-0)$ & $\mathrm{n}>10$ \\
Chèvres & $\mathrm{C}=150+80 \mathrm{n}$ & $\mathrm{n} \leqq 10$ \\
& $\mathrm{C}=950+40(\mathrm{n}-10)$ & $\mathrm{n}>10$ \\
\hline
\end{tabular}

$\mathrm{C}$ - capacité minimale de la pompe à vide en litres d'air atmosphérique par minute.

$\mathrm{n}=$ nombre de faisceaux-trayeurs (griffes).

vaches ne semblent pas être très bien établies. Il existe de nombreux cas où des capacités de pompe inférieures donnent entière satisfaction. Avec les vaches, on admet en général qu'un faisceau-trayeur nécessite approximativement $45 \mathrm{l} / \mathrm{min}$ (Doane et al 1980) à $60 \mathrm{l} / \mathrm{min}$ (Akam 1977). Aussi, selon Spencer (1982), une méthode réaliste pour définir la capacité minimale de la pompe à vide serait de définir une réserve de base pour la manipulation des faisceaux-trayeurs puis d'ajouter « 60 à $80 \mathrm{l} / \mathrm{min}$ par faisceau ». La norme française suit plus ou moins cette règle mais, on peut continuer à s'interroger sur la valeur à donner à la réserve de base.

\section{Conclusion}

Les recherches sur la traite sont concentrées sur la vache laitière qui représente une production largement prépondérante. Grâce aux travaux effectués notamment par l'INRA, on sait également conseiller l'éleveur qui souhaite traire des brebis laitières. Par contre, les chercheurs et les constructeurs de matériel ont accordé moins d'attention à la production caprine traditionnellement considérée comme marginale. Il existe peu de références et elles concernent surtout les races Saanen et Alpine. Il convient donc d'être prudent pour guider l'éleveur dans un choix raisonné. Pour minimiser les risques, il sera souvent conduit à adapter des solutions qui semblent satisfaisantes pour des conditions d'élevages similaires aux siennes. Elles peuvent être inapplicables si le contexte socio-économique est différent.

Que ce soit avec un simple quai de traite ou avec un manège de traite plus complexe, pour traire un grand nombre de chèvres à l'heure, il est indispensable d'avoir une technique de traite simple. On doit s'efforcer de traire sans lavage préalable du pis et sans égouttage après la traite car ces deux opérations sont les plus coûteuses en main-d'œuvre.

Pour diminuer la pénibilité du travail, on peut décaler les horaires des traites et il n'y a pas d'inconvénients à adopter des intervalles très inégaux entre elles en trayant par exemple à 8 heures et 16 heures.

S'il faut libérer la main-d'œuvre pour d'autres travaux, l'éleveur a la possibilité de réduire la fréquence des traites. En supprimant celle du dimanche soir ou en trayant une fois par jour vers la fin de la lactation, dans certaines conditions, les baisses de production peuvent être acceptables. Toutefois, il y a des répercussions importantes sur la production et la composition du lait. L'adoption non maîtrisée de telles pratiques présente des inconvénients majeurs non seulement pour la fabrication fromagère mais surtout pour le contrôle des performances lorsque les élevages adhèrent au contrôle laitier.

Il faut souligner que ces possibilités de diminuer les contraintes liées à la traite impliquent une bonne adaptation du matériel à la physiologie de l'animal. On considère en général que la chèvre est " facile " à traire. Or, dans certains troupeaux au moins, il existe des animaux qui semblent inaptes à la traite mécanique. En effet, les trayons sont terminés par des canaux qui s'ouvrent difficilement sous l'effet du vide créé par la machine (Le Du et Benmederbel, 1984). Actuellement, on dispose de peu d'informations sur la variabilité des caractères qui représenteraient "l'aptitude à la traite ». Sous quelques aspects, le matériel de traite est différent de celui qui est utilisé pour les vaches (manchon, griffe, pulsation, niveau de vide) bien qu'il soit difficile de justifier certains choix. Une norme française de 1985 spécifique aux chèvres représente toutefois une garantie pour l'éleveur en ce qui concerne la construction et les performances du matériel.

Ce texte a été élaboré d'après un rapport présenté à la 4 Conférence Internationale sur la chèvre qui s'est tenue à Brasilia, Brésil, du 8 au 13 mars 1987.

\section{Références bibliographiques}

AKAM D., 1977. Description and performance of components. In : THIEL C.C., DODD F.H. (Ed.), Machine milking, 45, N.I.R.D., Reading, Grande-Bretagne.

BOUILLON J., LE MENS P., 1984. Comparaison de différents manchons trayeurs chez la chèvre. In: Proc. 3 Symp. Int. Traite Mécanique Petits Ruminants, 16-20 mai 1983, Espagne. Ed. Sever Cuesta, Valladolid, Espagne, 479-481.

CASU S., CARTA R., 1974. Influence de la vitesse de pulsation sur l'efficacité de traite chez la brebis Sarde. In : Proc. $1^{\text {er }}$ Symp. Int. Traite Mécanique Petits Ruminants, 7-11 mai 1973, Millau, France, Ann. Zootech., numéro hors série, 201-203.

CICOGNA M., SANGIORGI F., 1984. Comparaisons des caractéristiques de traite de chèvre avec six types de faisceaux-trayeurs. In : Proc. $3^{\circ}$ Symp. Int. Traite Mécanique Petits Ruminants, 16-20 mai 1983, Valladolid, Espagne. Ed. Sever Cuesta, Valladolid, Espagne, 485 491.

DARRACQ J., LE MENS P., PERROT C., 1978. Caractéristiques des machines à traire les chèvres utilisées en France et leur contrôle en ferme. In : Proc. 2" Symp. Int. Traite Mécanique Petits Ruminants, 22-27 mai 1978, Alghero, Italie. Ed. F.N.O.C.L., Paris, 324-345.

DISSET R., 1974. Les différents systèmes de traite des chèves. Organisation du travail de la traite. In : Proc. 1" Symp. Int. Traite Mécanique Petits Ruminants, 7-11 mai 1973, Millau, France, Ann. Zootech., numéro hors série, 267-280. 
DOANE M. NATKE R.P. SCOT"I N R, DELWICHE M.J.. BRAY D.R., 1980. Air flow utilization in milking parlours. Cornell University, Ithaca, Etats-Unis, paper 80-3027 ASAE, $28 \mathrm{pp}$.

DODD F.H., GRIFFIN T.K., 1977. Milking routines. In : THIEL C.C., DODD F.H. (Ed.), Machine Milking, 179-200. N.I.R.D., Reading, Grande-Bretagne.

GRACHEV I.I., 1953. The cerebral cortex and lactation Zhum. Obshch. Biologie, 14, 333-348.

HENDERSON A.J., BLATCHFORD D.R., PLAKER M. 1983. The effect of milking thrice instead of twice daily on milk secretion of the goat. Quart. Journal of Exp. Physiol. and Cognate Medical Sciences, 68, 645-652.

ISO 5707, 1982. Milking machine installations. Construction and performance. $1^{\text {"' éd., }} 14$ pp, I.S.O., Londres.

LABUSSIFRE J.. COMBAUD J.F., DOUAIRE G., 1978. Effet des conditions de sevrage sur la production laitière et le comportement à la traite des brebis Préalpes du Sud. In: Proc. 2 "Symp. Int. Traite Mécanique Pelits Ruminants, 22-27 mai 1978, Alghero, Italie. Ed. F.N.O.C.L. Paris, 146-163.

LE DU J., 1985. Functional parameters affecting the efficiency of milking machines adapted to sheeps and goats 36th Annual Meeting of the European Ass. for Anim. Prod., Kallithea, Greece. Vol. 1, 430-431 (abstract).

LE DU I., 1987. Facilities and equipment for hand and machine milking of goats. In: Proc. IV Int. Conf. on Goats, 8-13 mars 1987. Brasilia, Brazil, Ed. EMBRAPA DPP, Doc. 14, 2 Vol. 269-282.

LE DU J. BENMEDERBEL B 1984. Aplitude des chèvres de race Saanen à la traite mécanique. Relations avec les caractéristiques physiques du trayon. Ann. Zootech.. 33 $375-384$

L.E DU J., LABUSSIERE J., PETREQUIN P., MIRMAN P., COMBAUD J.F. 1978. Effets de la pulsation, du mouvement du manchon et des conditions d'écoulement du lai sur la traite des brebis Préalpes du Sud. In : Proc. 2 Symp. Int. Traite Mécanique Petits Ruminants, 22-27 ma 1978, Alghero, Italie. Ed. F.N.O.C.L., Paris, 363-384.

LE JAOUEN J.C., 1981. Milking and the technolugr of milk and milk products. In : Goat Production. Ed. C. GALL, Academic Press, London, 345-377.

LE JAOUEN J.C., LE MENS P., SHELEAU J., 1984. Conception du chantier de traite caprin: proposition

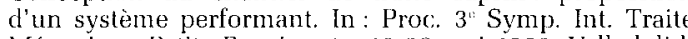
Mécanique Petits Ruminants, 16-20 mai 1983. Valladolid. Espagne. Ed. Sever Cuesta, Espagne, 357-361.

LE MENS P., 1977, Les manèges de traite des chèvres utilisés en France, 19 pp, Ed. S.P.E.O.C., Paris.
LE MLNS P., 1978. Suppression de la traite du dimanche soir chez la chèvre. Conséquences sur la production laitière et la composition du lait. In : Proc. 2" Symp. Int. Traite Mécanique Petits Ruminants, 22-27 mai 1978 , Alghero, Italie. Fd. F.N.O.C.L., Paris, 277-288.

LE MENS P., 1981. Traite mécanique des chèvres: le chantier de traite. incidence de la machine sur les caractéristiques de traite. $6^{\prime \prime}$ Journées de la Recherche Ovine et Caprine, Toulouse, France. Ed. SPEOC, Paris, 153-170.

LE MENS P., 1984. Analyse et conception du chantier de traile caprin. In : Proc. $3^{*}$ Symp. Int. Traite Mécanique Petits Ruminants, 16-20 mai 1983. Valladolid, Espagne. Ed. Sever Cuesta, Espagne, 347-356.

LE MENS P., DISSET R,, 1984. Comparaison de deux griffes à traire les chèvres. In : Proc. 3" Symp. Int. Traite Mécanique Petits Ruminants, 22-27 mai 1978, Valladolid, Espagne. Ed. Sever Cuesta, Valladolid, Espagne, 482-484. LE MENS P., TOUSSAINT G., LE JAOUEN J.C., 1978. Temps de travaux et systèmes de traite mécanique des chèvres en France. In : Proc. 2" Symp. Int. Traite Mécanique Pelits Ruminants, 22-27 mai 1978, Alghero, Italie. Ed. F.N.O.C.L., Paris, 289-305.

LE MENS P. BOULLON J., LAJOUS A., 1984. Comparaison du double vide par rapport au simple vide : Effet sur la vitesse de traite de chèvres de race Alpine Chamoisée et Saanen. In : Proc. 3" Symp. Int. Traite Mécanique Petits Ruminants, 22-27 mai 1978, Valladolid, Espagne. Ed. Sever Cuesta, Valladolid, Espagne, 468-472.

MOCQUOT J.C. 1978. Effets de l'omission ségulière et irrégulière d'une traite sur la production laitière de la chèvre.In : Proc. 2" Symp. Int Traite Mécanique Petits Ruminants, $22-27$ mai 1978 , Alghero, Italie. Ed. F.N.O.C.L.. Paris, 175-201

MOCQUOT J.C., 1980. La réduction du nombre de traites: mythe ou réalité. Chèvre, 121, 25-31.

RICORDEAU G., LABUSSIERE J., 1970. Traite à la machine des chèves. Comparaison de 2 rapports de pulsation et efficacité de la préparation de la mamelle avant la traite. Ann. Zootech., 19, 37-43.

SPENCER S.B., 1982. Sizing milking systems. A review, in "Proc. 21st An. Meeting Nat. Mastitis Council», Washington D.C., U.S.A., 141-149.

TVERSKOI G.B., RIMMA I.. NOVIKOVA O.P., JESTOKANO V., 1984. Influencia del ordeno mecanico sobre la generacion de impulsos nerviosos desde los receptous del pezon de la ubre de capra. In : Proc. 3" Symp. Int. Traite Petits Ruminants, $16-20$ mai 1983, Valladolid, Espagne. Ed. Sever Cuesta, Espagne, 87-99.

U 36-011, 1985. Installation de traite mécanique. Construction et performance, AFNOR, Paris, France, $37 \mathrm{p}$. 